\title{
The Protection and Inheritance of Suzhou Opera*
}

\author{
Guorong Ding \\ College of Art in SUST \\ Suzhou University of Science and Technology \\ Suzhou, China 215011
}

\begin{abstract}
Suzhou Opera is the first batch of national intangible cultural heritage of China. This paper attempts to explore the protection and inheritance of its heritage from three aspects: the consensus on the concept of "innovation", the role of inheritor as "human" and digital protection.
\end{abstract}

Keywords-Suzhou Opera; intangible cultural heritage; protection

\section{INTRODUCTION}

As a local opera in Suzhou, Suzhou Opera is a new type of drama formed in the early 1940s. In May 2006, China's first batch of national intangible cultural heritage list was promulgated, and Suzhou opera was included in the list (199IV-55).

The predecessor of the Suzhou opera was Sutan which was originally named Duibai Nanci, Kun Opera and Huagu Tanhuang. It is originally a mouthpiece-style opera singing by actor without makeup and acting. During the reign of Emperors Kangxi and Qianlong in the Qing Dynasty, Nanci and Tanhuang merged together and formed Suzhou Tanhuang. In the middle and late period of Qing Dynasty, Sutan became increasingly popular.

The Sutan performance with simplistic makeup appeared in the 1920s was the first step in the drama of Suzhou Opera. Later, in 1941, the Suzhou Opera Troupe with National Customs was established. In 1951, Minfeng Shiyan Suzhou Opera Troupe was established, marking the official formation of Suzhou Opera. The tune of Suzhou opera is beautiful, clear and mildly pleasant, and the dialogue Wunongruanyu is quite fascinating with local characteristics and flavor. In the 1950s, 1960s, and 1980s, Suzhou Opera set off three climaxes in Suzhou. In Suzhou region, Suzhou Opera is well-known and is spread all over China, becoming one of the major dramas in Jiangsu Province. At that time, for reason that Kun Opera was too high to be popular while Suzhou Opera was very popular, there was a phenomenon as the saying goes "Suzhou Opera is supported by Kun Opera in art, and Kun Opera is supported by Suzhou Opera in performance".

Suzhou Opera once was called one of the "three flowers in Suzhou art circle" together with Kun Opera and Pingtan (storytelling and ballad singing in Suzhou dialect). However,

*Fund: This paper is the research result of the Philosophy and Socia Science Fund Project "Study on Suzhou Opera from the Perspective of Intangible Cultural Heritage" (No.: 2017SJB1353) for Colleges and Universities in Jiangsu Province. after a short period of glory, Suzhou Opera gradually disappeared. Kun Opera was listed in the world's intangible cultural heritage list and attracted the attention of the world; Pingtan was granted the reputation of "the most beautiful voice in the world"; the two operas both have been included in specialized schools to provide relevant education and inherit it, while Suzhou Opera gradually fades out of people's vision.

But when looking at the evolution history of Suzhou Opera, it may be found that Suzhou Opera has rich cultural values and historical heritage values. From the perspective of tone, Suzhou Opera can be traced back to the Ming Dynasty and has more than three hundred years of history. It can be said that Suzhou Opera preserves a rich music heritage of local drama. Suzhou Opera is developed on the basis of Xuan Juan (宣卷), Tanci (弹词, storytelling to the accompaniment of stringed instruments), and rap music, and is adapted from folk mountainous songs and ditties. This music evolution process once played a certain role in the development of Shanghai Opera and Wuxi Opera. In terms of literary creation, full-scale dramas in the list of Suzhou operas are almost transplanted from the legend of Kun Opera. In a certain sense, Suzhou Opera is a popular version of Kun Opera. In terms of performance, Kun Opera has rich types of performance art and profound connotation. Suzhou Opera often learns from the performance art of Kun Opera so that the player can play both Kun Opera and Suzhou Opera, becoming amphibious players. The origin created the characteristics of Suzhou Opera that is different from other local operas.

The protection of intangible cultural heritage has become a hotspot vocabulary and action in recent years. On October 17, 2003, the 32nd Congress of UNESCO adopted the Convention for the Safeguarding of the Intangible Cultural Heritage, the most important document of United Nations on the protection of intangible cultural heritage. This Convention defines the intangible cultural heritage in detail:

"Intangible cultural heritage refers to the various social practices, conceptual expressions, expression forms, knowledge, skills and related tools, objects, handicrafts and cultural sites that are considered as a part of cultural heritage by the community groups and sometimes by individuals. This intangible cultural heritage is passed down from generation to generation, and has been continuously recreated in the interaction of communities and groups with the surrounding environment and with nature and history, 
irregular educations) and revitalization of such heritage from various perspective to maintain the vitality of intangible cultural heritage."

The protection of Suzhou Opera is a proposition on how to do it. This paper tries to put forward the following aspects to focus on the protection strategy of Suzhou Opera heritage to seek for joint discussion.

\section{The CONSENSUS ON THE CONCEPT OF "INNOVATION"}

Innovation is a unique cognitive ability and practical ability of human beings. It is a high-level expression form of human's subjective initiative and an inexhaustible motive force for driving the national progress and social development. Meanwhile, in the history of art development, innovation is of great significance. The development of art depends on the driving of innovation.

In 1942, a group of Yue opera actresses represented by Yuan Xuefen advocated "New Yue Opera" and further reformed Yue Opera. Under the participation and help of some intellectuals engaging in modern drama, film, literature and music, the original system without script but the outline was replaced by the system with script and director, and the traditional costumes, costumes and settings were reformed. In music, its tone also tends to be delicate and euphemistic. "Chidiao (尺调)" tone and the reversal tone "Xianxiadiao (弦 下调)" successively appear, making the tones of Yue Opera more and more expressive. While using traditional instrumental tunes as the scene music, the composer also once conducted "specialized songs for special purpose, theme development and simple multi-voice accompaniment attempts". In the compilation of the band, the Chinese and Western instruments are combined with each other, and even an attempt is made to play fully by western instruments. Eventually a form of accompaniment with full national instruments was formed in the late 1940s. The 1940s was a period of prosperous development of Yue Opera. In the prosperous performance activities, a number of actors gradually emerged due to their unique styles of singing, forming several different genres of singing, such as Yuan Xuefen's simple and euphemistic "Yuan-style" singing tune, Fu Quanxiang's ups-and-downs "Fu-style" singing tune, Qi Yaxian's sorrowful mourning "Qi-style" singing tune (all of which are sung by actresses), Yin Guifang's fluent and deep "Yin-style" singing tune, Xu Yulan's gorgeous "Xu-style" singing tune, and Fan Ruijuan's simple and mellow "Fanstyle" singing tune. With the development in art, Yue Opera has gradually become a very popular opera type in Shanghai, Jiangsu and Zhejiang region.

Yuan Xuefen said Yue Opera was early supported by Kun Opera and modern drama. By learning from Kun Opera, Yue Opera can learn the excellent drama tradition; by learning from modern drama, Yue Opera can take the lead in enabling the director system which is an innovation. In a certain sense, Yue Opera both inherits classics and makes innovative development.

From the development history of drama, a change in tone can promote the formation and development of a drama type. Wei Liangfu changes the Kunshan tone in the method of confirmation, filing, research, preserving, propagation, carrying forward, inheritance (mainly through regular and 
"formulating the rules and norms of tone on the basis of Suzhou accent, creating elegant tone and enriching the performance skills, establishing and gradually perfecting the scene of Kun Opera, integrating the southern and northern tones, and treating Kunshan tone as the formal tone" and forms a fluent and far-reaching new Kunshan tone, promoted the spreading of Kun Opera.

Intangible cultural heritage is a kind of dynamic legacy. It is the current evolution of human's practice process in the past, and the inheritance and development of living cultural skills and spirit. Therefore, the inheritors shoulder a dual mission: inheritance and creation. They should not only pass down the superb crafts and skills that they have mastered to future generations, but also continuously develop the knowledge and skills that they possess in the process of the inheritance, so that the inherited crafts and skills are enhanced by innovation and development.

In terms of the protection of Suzhou Opera and Kun Opera heritages, the author once interviewed Ms. Qian Ying, the former director of the Cultural Bureau. She believed that people generally had misunderstanding and misinterpretation of "innovation" at present. She said that there were two views: experts believed that the the focus should be on protection and rescuing, while governmental departments emphasized innovation and thought that the popularity and the way of its sustainability are dependent on innovation. In fact, this is a narrow understanding of innovation. It seems that when it comes to innovation, it is to launch new list of operas. In fact, innovation exists in inheritance.

\section{In the interview, Ms. Qian Ying said:}

"In fact, when managers go down to the grassroots level and watch the actors learning drama from the actor named with prefix of chuan (传), they are asked to process what they saw; the processing process is consolidation and getting rid of unnecessary things. ... Those new artistic workers very respect the actors named with prefix of chuan who are also making innovation and some dramas are created by them. Before the reform and opening up and even nowadays, I have always thought that Suzhou is conservative. I have been thinking about it for a few years. In fact, Suzhou is not conservative. Now it is recognized by people that only Suzhou Opera and Kun Opera maintain the characteristics of South Kun Opera mainly in singing and performance art and maintain the original script and form of Kun Opera. Therefore, innovation of Kun Opera can't transcend the artistic ontology of Kun Opera itself."

The views presented also apply to the protection of Suzhou Opera. That is to say, innovation cannot be made with the most fundamental things abandoned

Relevant scholars believe that innovation should be a very high standard for art, but now it has become a pretext for a group of shoddy and unpopular art.

"Innovation should be a tough transition to tradition through a lot of learning and imitation and a haunting glimpse on the basis of numerous imitations and repetitions of ordinary artists. But now it has become graffiti of ignorant child. Graffiti. ...but the development of art must be in stages at the same time. In order to transit from one stage to a new stage, innovation is surely needed; it is precisely large amount of imitation and repetition that composed the subject of each stage." 1

Suzhou Opera needs innovation. Its defect in selfdevelopment even more needs innovation and reform. Suzhou Opera has changed from simple singing without makeup and acting to stage-art form. Its tone is bound to be inadaptable, and in urgent need of innovation and improvement. Because simple singing without makeup and acting is not restricted by the action on stage, it does not place high requirements on the action and drama properties of the tone. It is always sung indoor in a venue not that large, with not that many audiences. This situation will definitely affect the direction and development of music. Therefore, the tunes of Suzhou Opera are more suitable for singing in a small voice, instead of loud singing. The process of Suzhou Opera going from the simple singing to dramatic music and further to the stage is still in its infancy, not reaching the peak of art. For example, declarative tune without ideal, when writing the script, wherever it is necessary to declare cannot be arranged with singing section but speaking.

The reason why opera can attract audience depends largely on the circulation of its tone. The attractiveness of Suzhou Opera also lies in its moving tone. Its tunes are wide and euphemistical. However, Suzhou Opera's tone has a wide range of sounds; it is neither easy to learn nor even to sing. So it is impossible to be widely sung and popular like Yue Opera and Huangmei Opera. Can it be adapted into a group of easy-to-sing tunes to facilitate the singing and popularization?

In the nearly half century since the formation of Suzhou Opera, many professional composers such as Jiang Shouliang, Wang Zhengnan, Zhang Fengyuan, and Jin Sha participated in the work, and worked together with Suzhou Opera artists Hua Hesheng, Zhu Xiaofeng, Zhuang Zaichun, Jiang Yufang, Yin Simin, Ding Jie and so on for long term, making great efforts to the inheritance and development of Suzhou Opera music. In order to adapt to new theme and style of script and the rhythm variation of the lyrics, the music of Suzhou Opera has been innovated. For example, "Liu Sanjie (刘三姐)" is a comedy of the folk song style composed by Jiang Shouliang on the basis of folk songs in Wuzhong region, and reflects the style of this comedy of folk song. For another example, the original lyric of "The Fifth Girl (五姑娘)" is a long narrative folk song of Shuzhou. In order to maintain the original lyrics, Jin Sha creatively adapted the [Shuban (数板)] and [Dieban (叠板)], so that the 34-character sentence sung between prison and Taihu lakeside can be freely expressed. This way full expresses the personality characteristics of the fifth girl.

The first repertoire after Minfeng Suzhou Opera Troupe was established, "The blood-stained plum blossom fan (血溅 桃花扇)", was directed by the famous director Ying Yunwei.

Fu Jin. Pouring a Pot of Cold Water toward "Innovation": A Conservative's Self-talk. Pass The Flame: The Theory and Practice of the Protection of Intangible Cultural Heritage. China Social Sciences Press, 2008, P98. 
Third, recording of their activities in tangible forms Compared with other local operas, Suzhou Opera used opera director system earlier. Hence, it can be seen that since the emergence of Suzhou Opera, it has become inseparable from innovation. It is precisely because of innovation that Suzhou Opera has revived to the previous glorious time from dying.

\section{THE ROLE OF INHERITOR AS "HUMAN"}

The inheritance of intangible cultural heritage is more complicated and more human-dependent than that of tangible cultural heritage. For intangible cultural heritage, the UNESCO's Guidelines for the Establishment of National "Living Human Treasures" Systems maintains that:

"Although the techniques of producing crafts and even the cooking techniques can be written down, the creation behavior actually has no material form. Performance and creation are intangible, and their skills and crafts only exist in those who engage in them." 2

Inheritance is the basic feature of intangible cultural heritage. Only through the oral communication of the "people who engage in them" can the intangible cultural heritage be passed down from generation to generation.

The inheritance of intangible cultural heritage is realized by the inheritors. The inheritors of intangible cultural heritage is a person or group of people who represent(s) the profound national and folk cultural tradition of a heritage, possess(es) such crafts, skills and abilities that are of significant value and can carry forward an intangible cultural heritage and is/are at the highest level. They are recognized by communities, groups, and ethnic groups. Inheritors are important carriers and transmitters of intangible cultural heritage. They create, master, and carry the cultural traditions and superb skills related to an intangible cultural heritage based on extraordinary intelligence and spirituality. The intangible cultural heritage is precisely carried forward by them. An outstanding inheritor is the representative of a certain class of intangible cultural heritage.

Intangible cultural heritage is a kind of dynamic legacy. It is the current evolution of human's previous practice process, and the inheritance and development of living cultural skills and spirit. Therefore, the inheritors carry a dual mission, namely inheritance and creation. For such inheritors, it is not only necessary to pass down the superb crafts and skills that they have mastered to future generations, but also necessary to continuously carry forward the knowledge and skills that they possess in the process of the inheritance, so that the inherited crafts and skills are improved through innovation and development.

With regard to the obligations of intangible cultural heritage inheritors, the Guidelines stipulates, "The obligations of "living human treasures" should be:

First, improving their knowledge and skills;

Second, imparting their knowledge and skills to the younger generations;

\footnotetext{
Wang Wenzhang. Introduction to Intangible Cultural Heritage. Educational Science Press, 2008, p258.
}

(video or audio recording, publications, etc.) without copyright issue and dispute;

Fourth, publishing to the public such products as produced by using their skills and techniques under normal conditions."

The Interim Measures for the Recognition and Management of Representatives of National Intangible Cultural Heritage Projects issued by China in 2008 is a management document relevant to China's first national intangible cultural heritage project, marking China's important progress in the construction of laws and regulations for intangible cultural heritage protection.

The status of Suzhou Opera inheritors is as follows:

There are currently 10 representative inheritors above the municipal level, including 3 national inheritors, 2 provincial inheritors and 5 municipal inheritors.

Wherein, Yin Siming and Jiang Yufang (deceased) are the second batch of national representative inheritors of intangible cultural heritage; Ding Jie (deceased) is the third batch of national representative inheritors of intangible cultural heritage.

Yin Jimei and Ling Jiqin are the third batch of provincial representative inheritors of intangible cultural heritage.

Wang Fang and Liu Jiyan are the first batch of municipal representative inheritors of intangible cultural heritage in Suzhou; Liang Qinqin, Jin Jiakun (deceased) and Pan Yuqin are the second batch of municipal representative inheritors of intangible cultural heritage in Suzhou.

At present, the status of personnel in the interitance system of Suzhou Opera is as follows:

- Inheritors: 3 national inheritors, 2 provincial inheritors, and 5 municipal inheritors. Some of them may occasionally engage in the teaching and performance of Suzhou Opera;

- The generation of people named with a prefix of "Ji (继)" and "Cheng (承)": more than 30 people; some of them occasionally engage in the teaching and performance of Suzhou Opera;

- The generation of people named with a prefix of "Hong (弘)": more than 10 people (including only one music composer), full-time engaged in Kun Opera;

- The generation of people named with a prefix of "Yang(扬)": about 20 people, full-time engaged in Kun Opera;

- New generation: 11 Suzhou Opera actors newly recruited and cultivated by Suzhou Wuxi Opera Troupe, specializing in Suzhou Opera. Since 2006, they has started to study Suzhou Opera and performed on stage.

Whether it is to rescue, protect, inherit or develop, it is inseparable from talents from generation to generation. 
Generally, it takes a small cycle and a large cycle for artistic performance talents to grow up and become mature respectively. The small cycle is about ten years, namely there should have been a batch of young top actors over ten years of cultivation. The large cycle is about twenty years, namely there should have been some mature famous actors over 20 years of cultivation.

Fortunately, the growth of oung actors of Suzhou Opera can be seen from the following aspects:

In 2009, the fourth Hongmei Cup in Jiangsu Province kicked off. The troupe leader arranged Wuxi Opera and Suzhou Opera actors both to take part in the match. Finally, Wuxi Opera actors won the third prize and Suzhou Opera actors won the second prize. In 2012, the first Hongmeipiaoxiang Themed Culture and Art festival of Jiangsu Province was held in Nanjing. I was fortunate enough to participate in the competition. The leader let me participate in competition through performing Suzhou Opera. This is a young drama for me, and my heart is extremely up and down. Later, I passed the semi-finals and finals and won the first Jiangsu "Stage Art Newcomer Award". Then, my Suzhou Opera drama “Jasmine Fragrance (莱莉飘香)” won the "Best Performance" award at the Binzhou Boxing Drama Art Festival held in Shandong. The achievements and honors in Suzhou Opera enhanced my confidence in learning and singing Suzhou Opera. ${ }^{3}$

Generally, as long as conditioned, it is possible to cultivate cast members of a drama; but it is difficult to cultivate the audience, especially for Suzhou Opera which has been no audience for 20 years. Since the mid-1980s, almost no one shows any interest in Suzhou Opera. Nowadays, the rehearsed Suzhou Opera repertoires are almost performed in public welfare activities. The real audiences of Suzhou Opera almost do not exist. In 2012, the author conducted a survey on Kun Opera in a university in Suzhou, and also investigated the students' understanding of Suzhou Opera. As a result, only several questionnaires among more than 200 questionnaires indicated that they had heard of Suzhou Opera. In today's society, even for Kun Opera, it is difficult to cultivate a rich and broad audience base, but it has a small group of "fans" whose existence can make the ancient Kun Opera "revive" but cannot achieve "prosperity". However, for many local dramas, they even do not have fans and thus are difficult to revive. Therefore, for Suzhou Opera without market and audience, how to develop becomes the issue worthy of consideration the most.

\section{Digital PROTECTION}

"If there is no research on Shanghai's old record, we may have forgotten Sutan which was once the second drama type of Shanghai, and its contribution to Shanghai culture," Qian Nairong said. "Sutan was popular in Shanghai at the beginning of 20th century. Due to the emergence of recording industry, many precious Sutan performances were

Yang Minhua. From Wuxi Opera to Suzhou Opera. Drama and Film Journal, No. 6, 2012, p102.

4 http://bbs.news.163.com/bbs/society/342662181.html recorded and further collected and protected by people of insight.

With regard to the obligations of intangible cultural heritage inheritors, the Guidelines stipulates, "the 4th obligation of Human Living Treasures" is to record their activities in tangible forms (video or audio recording, publications, etc.) without copyright issue and dispute.

From 1961 to 1962, Zhou Enlai and Chen Yi led the adjustment of cultural front, and put forward many important opinions on the protection and rescue of cultural and artistic heritage and the carrying forward of national traditional art. Chen Yi advocated that the party and the government should spare no effort to rescue traditional culture and art, and proposed specific measures to rescue and protect cultural and artistic heritage:

Many old dramas are endangered in China and must be rescued. ... Produce hundreds and tens of thousands of books to record traditional repertoire and traditional performance art, and take relevant pictures. Even if it is a dross, recording should go first. A lot of texts, scripts and photos should be saved, stored as materials, copied, and reprinted.

In April 1986, Jiangsu Provincial Institute of Literature and Art and Suzhou Drama Research Office specially collected all the old artists together and held a Sutan singing activity in Heyuan, Hanjiaxiang, Suzhou city, in order to reproduce the scene of Sutan singing in the past. However, Yin Suming, an inheritor of Suzhou Opera, deeply regrets the inaction after the recording event:

"The activity scene was taken photos and record; but it's a pity that after the event, we never listened to the audio record nor saw the video, let alone providing us the audio and video record for memory (the organization originally promised to provide each of us a copy of the record). At the moment, what come into my mind is that when the Drama Expo Pavilions were open, Suzhou Opera, Kun Opera and Pingtan were respectively exhibited in three pavilions. Later, Suzhou Opera pavilion disappeared without any reason and the material about the "Reproduction of Sutan Singing Activity" in 1986 also disappeared. This makes us very sad, and also very regretful. The Activity organized in 1986 was very precious. Those who attended the activity include three old artists in our troupe and 8 external old artists; at that time, we were all about 70 years old. It can be called a hard-won grand meeting. ... It's impossible to meet again! We feel deeply regretful!" 5

The rapid development of digital media and networks today provides a digital perspective for the protection of cultural heritage. Currently in China and foreign countries, the development trend of digital protection of cultural heritage mainly involves the following fields: digital record, digital extraction, digital restoration, virtual heritage, digital reproduction and digital integration and so on. Combined with the above digital protection methods, it is suggested to

\footnotetext{
Chen Yi. Speech at the Symposium on the Compilation of Traditional Chinese Opera.
} 
as movies, television, internet, mobile phones, etc. has changed the behavior of people's social interactions and changed their cultural consumption methods.

\section{CONCLUSION}

The protection of intangible cultural heritage of operas is a complex and grand project that requires national will, social attention and personal participation in the construction. The road to rejuvenation of Chinese operas is closely combined with the comprehensive rejuvenation of Chinese traditional culture. If people can recognize this point, the revival of Suzhou's local opera, Suzhou Opera, is still hopeful. However, in addition to the focus and support of the external environment, constant development and enrichment of the unique artistic treasure trove of Suzhou Opera becomes a vital important thing to do.

\section{REFERENCES}

[1] Fu Jin. Pouring a Pot of Cold Water toward "Innovation": A Conservative's Self-talk. Pass The Flame: The Theory and Practice of the Protection of Intangible Cultural Heritage. China Social Sciences Press, 2008, P98. (in Chinese)

[2] Wang Wenzhang. Introduction to Intangible Cultural Heritage. Educational Science Press, 2008, p258. (in Chinese)

[3] Yang Minhua. From Wuxi Opera to Suzhou Opera. Drama and Film Journal, No. 6, 2012, p102. (in Chinese)

[4] http://bbs.news.163.com/bbs/society/342662181.html

[5] Chen Yi. Speech at the Symposium on the Compilation of Traditional Chinese Opera. (in Chinese)

\section{information of Suzhou Opera.}

\section{Virtual Heritage}

Virtual reality technology can be used for introducing the heritage of Suzhou Opera, such as the virtual Su Opera Museum project.

\section{Digital Reproduction}

According to archaeological findings, literature records or research conclusions, two-dimensional three-dimensional animation technology and augmented reality technology can be used for reproducing and interpreting a historical event or process in the development process of Suzhou Opera, such as reproducing the scene of "Sutan singing" and "Sutan makeup" to practically reproduce the endangered operas.

\section{E. Digital Integration}

Digital library (literature materials) and digital museum (national heritage books) are important physical platforms for cultural protection, communication and display, and are the two most typical applications based on digital integration. It is an organized warehouse with massive digital object information and knowledge and a distributed knowledge environment, integrated with collection and service, and can provide global information services based on the Internet. You can imagine a virtual theater program relevant to Suzhou Opera or other more opera arts...

The ever-decreasing drama audience has become an indisputable fact in today's world. The same is true of Chinese operas. Just as "each generation has its prevailing thing" ("Yi Yu Yue Lu" by Jiao Xun), the rise of media such 